\title{
AVALIAÇ̃̃O DA DEFICIÊNCIA DE NITROGÊNIO NO FEIJOEIRO USANDO UM MEDIDOR PORTÁTIL DE CLOROFILA
}

Murilo Mesquita Baesso ${ }^{1}$, Francisco de Assis de Carvalho Pinto², Daniel Marçal de Queiroz ${ }^{3}$, Nerilson Terra Santos ${ }^{4}$, José Eustáquio de Souza Carneiro

\section{RESUMO}

O nitrogênio $(\mathrm{N})$ é um dos principais nutrientes para o feijoeiro, sendo sua deficiência bastante comum. Como o nitrogênio também participa da constituição da molécula de clorofila, a avaliação da deficiência de $\mathrm{N}$ pela planta pode ser determinada pela mensuração indireta do teor de clorofila. Este trabalho teve como objetivo avaliar a possibilidade de utilização do medidor de clorofila na determinação da deficiência de N. O trabalho teve 5 tratamentos $(0,50,100$, 150 e $200 \mathrm{~kg} \mathrm{ha}^{-1} \mathrm{de} \mathrm{N}$ ), com 10 repetições, totalizando 50 vasos com $8 \mathrm{dm}^{3}$ de material de solo. Foram feitas 10 leituras por vaso aos 30, 40 e 50 DAE (dias após emergência). Os coeficientes de regressão linear simples e de segundo grau foram significativos para todas as épocas estudadas neste trabalho, entretanto, a falta de ajuste também foi significativa, exceto para o comportamento dos valores obtidos aos 50 DAE. De modo geral, os valores obtidos com o medidor de clorofila tenderam a aumentar com o acréscimo da quantidade de $\mathrm{N}$ aplicado e com o avanço do estádio de desenvolvimento da planta, indicando o potencial da utilização deste equipamento para discriminar as diferentes doses de nitrogênio aplicadas. Foi possível observar que as diferentes doses de nitrogênio aplicadas influenciaram os valores do medidor de clorofila.

Palavras-chave: agricultura de precisão, manejo nutricional e Phaseolus vulgaris.

\section{ABSTRACT \\ EVALUATION OF NITROGEN DEFICIENCY IN THE BEAN PLANT USING A PORTABLE CHLOROHYLL METER}

Nitrogen is one of the main nutrients for the bean plant, and its deficiency is very common. Because nitrogen also participates in construction of chlorophyll molecules, evaluation of $\mathrm{n}$ deficiency in the plant may be determined by indirect measurement of the chlorophyll content. The objective of this study was to use a chlorophyll meter for the detection of nitrogen deficiency. The study included 5 treatments $\left(0,50,100,150\right.$ and $200 \mathrm{~kg} \mathrm{ha}^{-1}$ of N), with 10 repetitions, summing 50 pots with $8 \mathrm{dm}^{3}$ of soil material. Ten readings were performed per pot at 30, 40 and 50 dae (days after emergency). The simple linear regression coefficients and those of second order were significant for all the study periods of this work, however the lack-of-fit was also significant, except for the behavior of the values obtained at 50 dae. In general, the values obtained with the chlorophyll meter trended to increase with elevation of $\mathrm{n}$ and with the advance of the plant development stages, indicating the potential for using this equipment to differentiate the various doses of nitrogen applied. It was verified that the different nitrogen doses applied influenced the values measured by the chlorophyll meter.

Keywords: precision farming, nutrient management and Phaseolus vulgaris

\section{Recebido para publicação em 25/08/2011. Aprovado em 01/02/2013.}

1 - Engenheiro Agrônomo, Professor, Faculdade de Zootecnia e Engenharia de Alimentos/ Pirassununga- SP, baesso@usp.br

2 - Engenheiro Agrícola, Professor, Universidade Federal de Viçosa/ Viçosa - MG, facpinto@ufv.br

3 - Engenheiro Agrícola, Professor, Universidade Federal de Viçosa/Viçosa - MG, e-mail: queiroz@ufv.br

4 - Engenheiro Agrônomo, Professor, Universidade Federal de Viçosa/ Viçosa - MG, e-mail: nsantos@ufv.br

5 - Engenheiro Agrônomo, Professor, Universidade Federal de Viçosa/Viçosa - MG, e-mail: jesc@ufv.br

\section{REVENG}




\section{INTRODUÇÃO}

$\mathrm{Na}$ cultura do feijoeiro, o nitrogênio é o macronutriente mais absorvido (ROSOLEM; MARUBAYASHI, 1994), estimulando o crescimento vegetativo da planta. Apesar de ser uma leguminosa, o $\mathrm{N}$ obtido pela fixação simbiótica não é suficiente para as plantas atingirem altos rendimentos. Além disso, sua aplicação estimula a absorção dos demais nutrientes.

Em aplicações convencionais de fertilizantes, a área é considerada homogênea, ou seja, a necessidade de adubo é tida como igual para toda a área, o que na prática não ocorre. Baseando-se nas necessidades médias da cultura, a aplicação ocorre de forma excessiva em algumas áreas do campo e insuficiente em outras, comprometendo a eficiência do processo de produção. Nesses casos, a agricultura de precisão é uma alternativa ao sistema convencional, com uma aplicação de nutrientes em quantidades variadas.

A análise foliar como método para determinação da deficiência de nitrogênio é bem precisa, existindo, porém, a limitação do custo elevado e da exigência de tempo para a coleta da amostra e sua análise. Exige ainda um grande número de amostras para que seus resultados sejam aplicados com sistemas de manejo localizado. A taxa de amostragem, demandada pelas técnicas de agricultura de precisão, pode inviabilizar economicamente sua adoção. Como o nitrogênio também participa da constituição da molécula de clorofila, a avaliação da necessidade de $\mathrm{N}$ pela planta pode ser determinada pela mensuração indireta do teor de clorofila (MALAVOLTA et al., 1997).

Os métodos geralmente utilizados para determinar a quantidade de clorofila na folha requerem destruição de amostras de tecido e muito trabalho no processo de sua obtenção. $\mathrm{O}$ desenvolvimento do medidor portátil de clorofila, permitindo leituras instantâneas do teor relativo de clorofila na folha sem destruí-la, surgiu como uma nova técnica para estimar esse teor (GILBER et al., 2001).

$O$ medidor de clorofila Minolta SPAD502 caracteriza-se pela rapidez, simplicidade e, principalmente, por possibilitar uma avaliação não destrutiva do tecido foliar. A determinação do teor de clorofila pelo clorofilômetro apresenta algumas vantagens sobre o método de extração de clorofila. Dentre essas, destacam-se: o resultado imediato do teor de clorofila na folha e o fato de o aparelho ter custo baixíssimo de manutenção (PIEKIELEK; FOX, 1992); a dispensa do envio de amostras para o laboratório, com economia de tempo e dinheiro; e a preparação de quantas amostras forem necessárias sem a destruição das folhas (MALAVOLTA et al., 1997).

Este trabalho teve como objetivo avaliar a possibilidade de utilização do medidor de clorofila na determinação da deficiência de $\mathrm{N}$.

\section{MATERIAL E MÉTODOS}

O trabalho foi conduzido em casa de vegetação, na cidade de Viçosa, Minas Gerais, localizada a uma altitude de aproximadamente $650 \mathrm{~m}$, com coordenadas geográficas de $20^{\circ} 45^{\prime} 54^{\prime \prime}$ latitude sul e $45^{\circ} 52^{\prime} 54$ " de longitude oeste. O trabalho teve cinco tratamentos $\left(0,50,100,150\right.$ e $200 \mathrm{~kg} \mathrm{ha}^{-1} \mathrm{de}$ $\mathrm{N})$, com 10 repetições, totalizando 50 vasos com oito $\mathrm{dm}^{3}$ de material de solo retirado do horizonte C, classificado como Latossolo Vermelho Distrófico. Os vasos foram dispostos em um delineamento inteiramente casualizado, em parcelas subdivididas no tempo: as doses foram alocadas nas parcelas e as épocas, nas subparcelas. $\mathrm{O}$ cultivo foi feito em vasos para que houvesse um melhor controle das quantidades aplicadas de nutrientes. Cada vaso teve três plantas de feijão (Phaseolus vulgaris) da cultivar BRSMG Talismã. Sendo o nitrogênio um nutriente de alta mobilidade no solo, a dose foi aplicada em duas etapas: um terço do total no ato do plantio e o restante aos 20 dias após a emergência (DAE). Os demais nutrientes foram misturados de maneira uniforme em todos os vasos com o material de solo, de acordo com os resultados da análise do solo, ficando apenas o nitrogênio como limitante na produção do feijão. Foi feita uma irrigação diária para que não houvesse nenhuma influência do déficit hídrico nas plantas. Para validação do nível de deficiência de nitrogênio na planta, foram feitas análises foliares em todos os vasos de cada tratamento aos 50 DAE.

No Quadro 1, estão mostradas as fontes de nutrientes e suas respectivas doses colocadas em cada vaso. Primeiramente foi posta uma dose de $5,44 \mathrm{~g}$ de calcário por vaso. Após isso, os vasos foram irrigados por 10 dias. Em uma segunda etapa, foi adicionada uma mistura de $2,19 \mathrm{~g}$ de cloreto de potássio, 3,2 $\mathrm{g}$ de superfosfato simples e $6,9 \mathrm{~g}$ de superfosfato triplo por vaso. Foram semeadas cinco sementes de feijão por vaso. Aos $20 \mathrm{DAE}$, procedeuse ao desbaste, deixando-se três plantas por vaso. 
Quadro 1. Fonte e nutriente por vaso

\begin{tabular}{|c|c|c|c|}
\hline Nutriente & Solo original & Fonte & Solo adubado \\
\hline Calcário & $\mathrm{pH} 5,0$ & $\begin{array}{l}\text { Mistura 4:1 de Cloreto de } \\
\text { cálcio e Cloreto de magnésio }\end{array}$ & $\mathrm{pH} 6,0$ \\
\hline Potássio & $18 \mathrm{mg} \mathrm{dm}^{-3}$ & Cloreto de potássio & $150 \mathrm{mg} \mathrm{dm}^{-3}$ \\
\hline Enxofre & $0,5 \mathrm{mg} \mathrm{dm}^{-3}$ & Superfosfato simples & $40 \mathrm{mg} \mathrm{dm}^{-3}$ \\
\hline Fósforo & $0,5 \mathrm{mg} \mathrm{dm}^{-3}$ & $\begin{array}{l}\text { Superfosfato simples e } \\
\text { Superfosfato triplo }\end{array}$ & $200 \mathrm{mg} \mathrm{dm}^{-3}$ \\
\hline Nitrogênio & - & Uréia & $0,50,100,150$ e $200 \mathrm{~kg} \mathrm{ha}^{-1}$ de $\mathrm{N}$ \\
\hline
\end{tabular}

Foi utilizado o medidor de clorofila SPAD 502 (Minolta Câmera Co, Ltd., Osaka, Japan). Foram realizadas 10 leituras por vaso, aos 30,40 e 50 DAE. As leituras foram tomadas sempre nas folhas jovens completamente expandidas. Foram testados o modelo de regressão linear simples, de $2^{\circ}$ grau e a falta de ajuste para os valores SPAD e as doses de nitrogênio aos 30, 40 e 50 DAE. Também foram feitas análises foliares aos $50 \mathrm{DAE}$, coletando-se cinco folhas. O método utilizado para determinação do $\mathrm{N}$ na folha foi o de Kjeldahl.

\section{RESULTADOS E DISCUSSÃO}

O coeficiente de correlação estimado entre a análise de $\mathrm{N}$ nas folhas e os valores SPAD obtidos aos 50 DAE foi de $0,72(\mathrm{P}<0,0004)$. Resultados semelhantes foram encontrados por Furlani Júnior et al. (1996), com uma correlação positiva entre as leituras do aparelho SPAD aos 40 DAE e os teores de $\mathrm{N}$ nas folhas do feijoeiro $(\mathrm{R}=0,75)$. Desta forma, pode-se inferir que a concentração de clorofila correlacionou-se positivamente com $\mathrm{o}$ teor de $\mathrm{N}$ nas folhas, representando o medidor portátil de clorofila SPAD-502, uma alternativa para determinar o status do nitrogênio no feijoeiro.

O modelo de regressão linear simples foi testado para os valores SPAD obtidos aos 30 DAE (Tabela 2), apesar de ter sido significativo $(\mathrm{P}<0,05)$, a falta de ajuste também foi significativa $(\mathrm{P}<0,05)$. A falta de ajuste significativa indica que o modelo linear simples pode não estar explicando a maior proporção da variação dos valores devido às diferentes doses de nitrogênio aplicadas.

Da mesma forma que o modelo de regressão linear simples, o de $2^{\circ}$ grau e a falta de ajuste foram significativos $(\mathrm{P}<0,05)$, (Tabela 3$)$. Segundo Regazzi (2000), no caso de resultado significativo para a falta de ajustamento nos modelos testados, deve-se escolher o modelo por meio de outros critérios, tais como: coeficiente de determinação $\left(\mathrm{R}^{2}\right)$, análise de resíduos e ponto de vista prático, entre outros. Segundo Martinez et al. (1999), a relação entre o crescimento da planta e a produção da cultura e o teor de nutrientes em seus tecidos tem uma curva dividida em cinco regiões: a primeira e a segunda definidas como região de deficiência, a terceira tida como de adequação, a quarta como absorção de luxo e a quinta como região de toxidez, caracterizada pelo decréscimo no crescimento e na produção. Sendo assim, o comportamento quadrático obtido pode ser explicado devido à dose de $200 \mathrm{~kg}$. ha- ${ }^{-1}$ de nitrogênio ter sido tóxica ao feijoeiro.

Observando o comportamento dos valores SPAD obtidos aos 30 DAE (Figura 1), percebecese uma tendência de aumento nos valores do SPAD até a dose de $150 \mathrm{~kg}$. ha ${ }^{-1}$ de nitrogênio, tendendo a decrescer na dose de $200 \mathrm{~kg}$. ha ${ }^{-1}$ de nitrogênio, o que demonstra um comportamento quadrático. $\mathrm{O}$ $\mathrm{R}^{2}$ da equação de $2^{\circ}$ grau foi maior, indicando que a regressão ajustada explica a maior proporção da variação devido às diferentes doses de nitrogênio, além de ser um comportamento agronomicamente esperado. Isso pode ser explicado devido ao aumento gradual no consumo de nitrogênio pelo feijoeiro até a fase do florescimento, após o início do florescimento há um decréscimo no consumo desse nutriente em virtude da diminuição da atividade metabólica radicular. Vale salientar que, em trabalho semelhante, o ajuste de $2^{\circ}$ grau também foi encontrado por Silveira et al. (2003).

\section{REVENG}


AVALIAÇÃO DA DEFICIÊNCIA DE NITROGÊNIO NO FEIJOEIRO USANDO UM MEDIDOR PORTÁTIL...

Quadro 2. Resultado da análise da regressão linear simples dos valores SPAD obtidos aos $30 \mathrm{DAE}$

\begin{tabular}{ccccc}
\hline FV & GL & SQ & QM & Valor-P \\
\hline Regressão & 1 & 895,8049 & 895,8049 & 0,0001 \\
Falta de ajuste & 3 & 313,0523 & 104,3508 & 0,0001 \\
\hline (Doses) & 4 & 1208,8572 & & \\
Resíduo & 45 & 71,5100 & 1,5891 & \\
\hline Total & 49 & 1280,3672 & & \\
\hline
\end{tabular}

$\mathrm{FV}=$ fator variável; $\mathrm{GL}=$ grau de liberdade; $\mathrm{SQ}=$ soma de quadrados; $\mathrm{QM}=$ quadrado médio; Valor $\mathrm{P}=$ significância.

Quadro 3. Resultado da análise da regressão linear de $2^{\circ}$ grau dos valores SPAD obtidos aos 30 DAE

\begin{tabular}{ccccc}
\hline FV & GL & SQ & QM & Valor-P \\
\hline Regressão & 2 & 1166,5770 & 583,2885 & 0,0000 \\
Falta de ajuste & 2 & 42,2802 & 21,1401 & 0,0000 \\
\hline (Doses) & 4 & 1208,8572 & & \\
Resíduo & 45 & 71,5100 & 1,5891 & \\
\hline Total & 49 & 1280,3672 & & \\
\hline
\end{tabular}

$\mathrm{FV}=$ fator variável; $\mathrm{GL}=$ grau de liberdade; $\mathrm{SQ}=$ soma de quadrados; $\mathrm{QM}=$ quadrado médio; Valor $\mathrm{P}=$ significância.

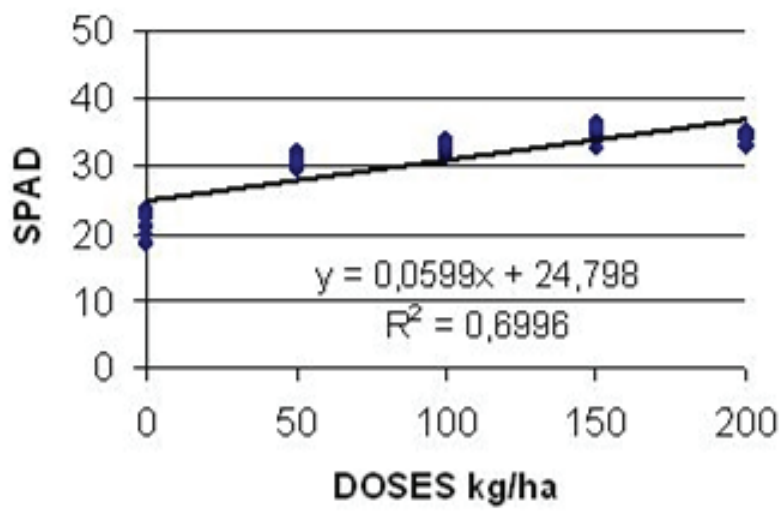

(a)

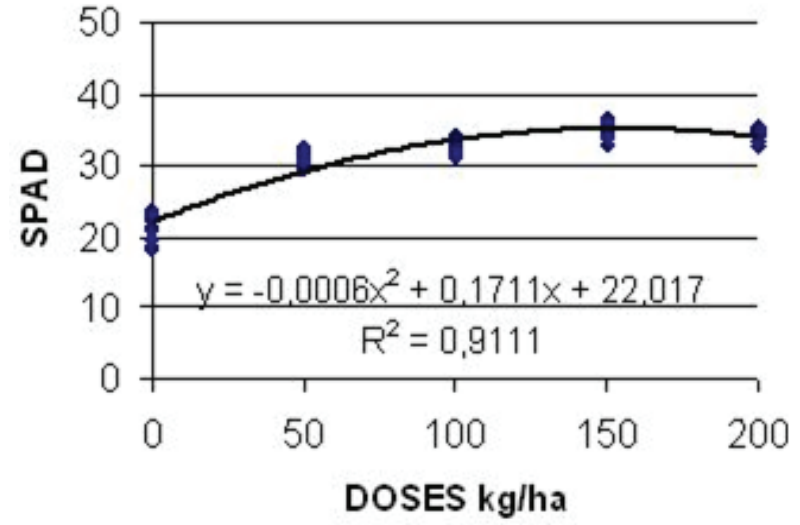

(b)

Figura 1. Regressão linear simples (a) e linear de $2^{\circ}$ grau (b) ajustadas para os valores SPAD obtidos aos 30 DAE em função das doses de nitrogênio.

O modelo de regressão linear simples foi testado para os valores SPAD obtidos aos 40 DAE (Quadro 4), apesar de ser significativo ( $\mathrm{P}<0,05)$, da mesma forma dos valores obtidos aos 30 DAE, a falta de ajuste também foi significativa $(\mathrm{P}<0,05)$.

$\mathrm{O}$ mesmo resultado foi apresentado pela regressão linear de $2^{\circ}$ grau (Quadro 5).

Aos $40 \mathrm{DAE}$, o $\mathrm{R}^{2}$ foi maior na regressão de $2^{\circ}$ grau ajustada (Figura 2). Os valores SPAD obtidos aos 40 DAE tenderam a aumentar na medida em que as doses de nitrogênio eram aumentadas, porém, é importante ressaltar que, acima dos $150 \mathrm{~kg}$. ha ${ }^{-1}$ de nitrogênio, os valores SPAD tenderam a se estabilizar. Isto indica que, nessa faixa, aumentandose a concentração do nutriente, o crescimento não é afetado, representando então o que se chama de "consumo de luxo" do elemento, definido como sendo o armazenamento do $\mathrm{N}$ no vacúolo em vez da sua participação na molécula de clorofila. O "consumo de luxo" nem sempre é indesejável, na medida em que permite à planta acumular nutrientes quando a disponibilidade deles é alta, liberando-os gradualmente, quando a absorção é insuficiente para suportar o crescimento. 
Quadro 4. Resultado da análise da regressão linear simples dos valores SPAD obtidos aos 40 DAE

\begin{tabular}{ccccc}
\hline FV & GL & SQ & QM & Valor-P \\
\hline Regressão & 1 & 733,8681 & 733,8681 & 0,0000 \\
Falta de ajuste & 3 & 180,8587 & 60,2862 & 0,0000 \\
\hline (Doses) & 4 & 914,7268 & & \\
Resíduo & 45 & 128,1620 & 2,8480 & \\
\hline Total & 49 & 1042,8888 & & \\
\hline
\end{tabular}

$\mathrm{FV}=$ fator variável; $\mathrm{GL}=$ grau de liberdade; $\mathrm{SQ}=$ soma de quadrados; $\mathrm{QM}=$ quadrado médio; Valor $\mathrm{P}=$ significância.

Quadro 5. Resultado da análise da regressão linear de $2^{\circ}$ grau dos valores SPAD obtidos aos 40 DAE

\begin{tabular}{ccccc}
\hline FV & GL & SQ & QM & Valor-P \\
\hline Regressão & 2 & 864,6259 & 432,3129 & 0,0000 \\
Falta de ajuste & 2 & 50,1009 & 25,0505 & 0,0006 \\
\hline (Doses) & 4 & 914,7268 & & \\
Resíduo & 45 & 128,1620 & 2,8480 & \\
\hline Total & 49 & 1042,8888 & & \\
\hline
\end{tabular}

$\mathrm{FV}=$ fator variável; $\mathrm{GL}=$ grau de liberdade; $\mathrm{SQ}=$ soma de quadrados; $\mathrm{QM}=$ quadrado médio; Valor $\mathrm{P}=$ significância.

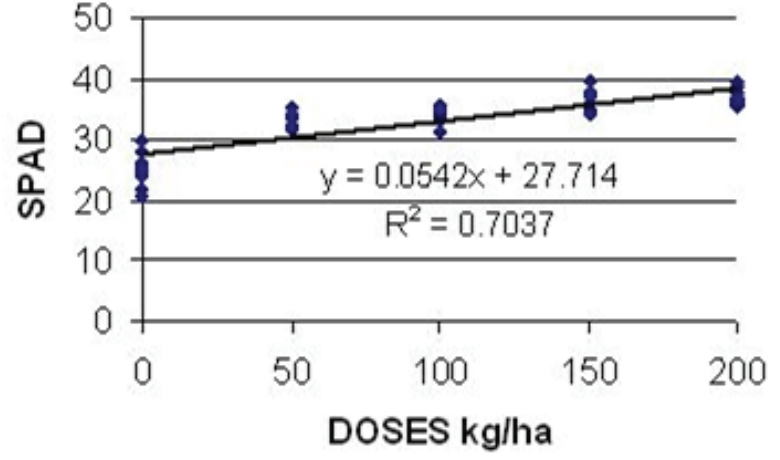

(a)

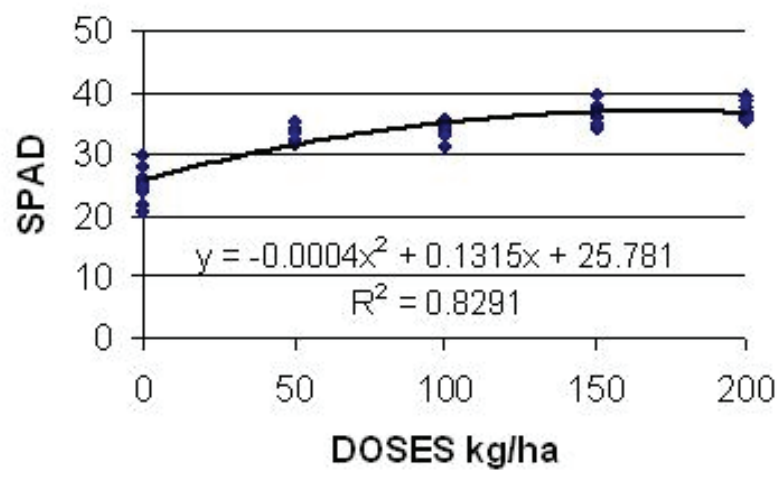

(b)

Figura 2. Regressão linear simples (a) e linear de $2^{\circ}$ grau (b) ajustadas para os valores SPAD obtidos aos 40 DAE em função das doses de nitrogênio.

O modelo de regressão linear simples foi testado para os valores SPAD obtidos aos 50 DAE (Quadro 6), apesar de ser significativo $(\mathrm{P}<0,05)$, a falta de ajuste também foi significativa $(\mathrm{P}<0,05)$.

Conforme resultado apresentado no Quadro 7, o modelo linear de $2^{\circ}$ grau (quadrático) foi significativo $(\mathrm{P}<0,05)$, já a falta de ajuste não foi significativa $(\mathrm{P}<0,05)$, indicando que o modelo linear de $2^{\circ}$ grau se ajusta à maior proporção da variação dos valores devido às diferentes doses de nitrogênio.

O comportamento dos valores SPAD obtidos aos 50 DAE pode ser observado na Figura 3. 
AVALIAÇÃO DA DEFICIÊNCIA DE NITROGÊNIO NO FEIJOEIRO USANDO UM MEDIDOR PORTÁTIL...

Quadro 6. Resultado da análise da regressão linear simples dos valores SPAD obtidos aos 50 DAE

\begin{tabular}{ccccc}
\hline FV & GL & SQ & QM & Valor-P \\
\hline Regressão & 1 & 573,6025 & 573,6025 & 0,0000 \\
Falta de ajuste & 3 & 56,6323 & 18,8774 & 0,0000 \\
\hline (Doses) & 4 & 630,2348 & & \\
Resíduo & 45 & 64,7550 & 1,4390 & \\
\hline Total & 49 & 694,9898 & & \\
\hline
\end{tabular}

$\mathrm{FV}=$ fator variável; $\mathrm{GL}=$ grau de liberdade; $\mathrm{SQ}=$ soma de quadrados; $\mathrm{QM}=$ quadrado médio; Valor $\mathrm{P}=$ significância.

Quadro 7. Resultado da análise da regressão linear de $2^{\circ}$ grau dos valores SPAD obtidos aos 50 DAE

\begin{tabular}{ccccc}
\hline FV & GL & SQ & QM & Valor-P \\
\hline Regressão & 2 & 622,6911 & 311,3456 & 0,0000 \\
Falta de ajuste & 2 & 7,5437 & 3,7718 & 0,0836 \\
\hline (Doses) & 4 & 630,2348 & & \\
Resíduo & 45 & 64,7550 & 1,4390 & \\
\hline Total & 49 & 694,9898 & & \\
\hline
\end{tabular}

$\mathrm{FV}=$ fator variável; $\mathrm{GL}=$ grau de liberdade; $\mathrm{SQ}=$ soma de quadrados; $\mathrm{QM}=$ quadrado médio; Valor $\mathrm{P}=$ significância.

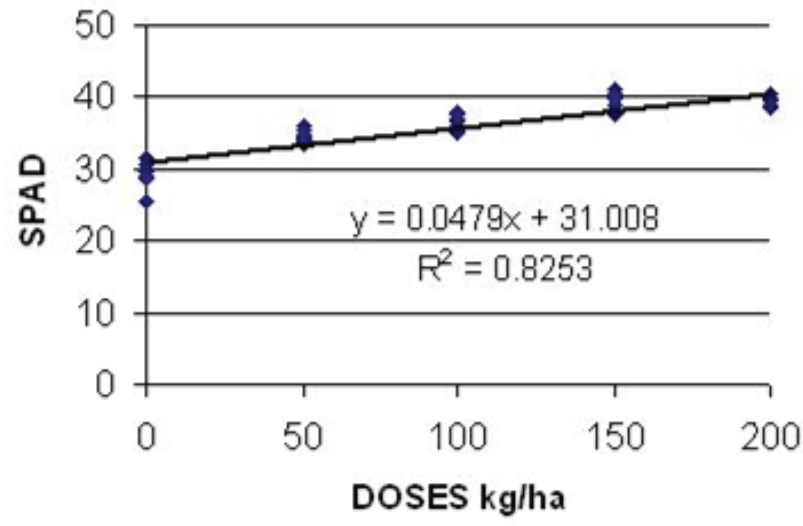

(a)

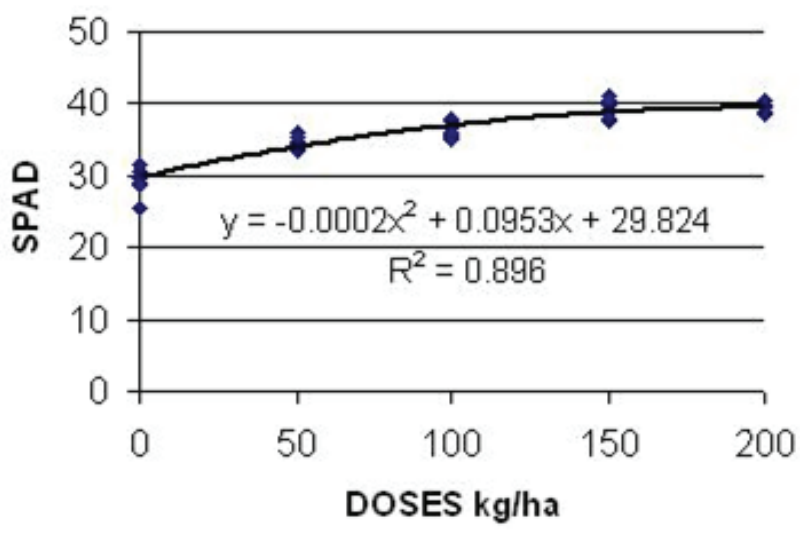

(b)

Figura 3. Regressão linear simples (a) e linear de $2^{\circ}$ grau (b) ajustadas para os valores SPAD obtidos aos 50 DAE em função das doses de nitrogênio.

\section{CONCLUSÕES}

Neste trabalho, foram testadas técnicas para identificação do status da deficiência de nitrogênio no feijoeiro utilizando um medidor de clorofila.

Nas condições em que o trabalho foi realizado, concluiu-se que:

- Foi encontrada uma correlação positiva entre análise de $\mathrm{N}$ nas folhas e os valores do medidor de clorofila, possibilitando a utilização do medidor de clorofila na determinação da deficiência de $\mathrm{N}$;

- O medidor de clorofila SPAD foi capaz de discriminar as doses de $\mathrm{N}$ aplicadas em todos os estádios avaliados, sendo, portanto, capaz de dizer quando está ocorrendo deficiência. 


\section{REFERÊNCIAS BIBLIOGRÁFICAS:}

FURLANI JÚNIOR, E.; NAKAGAWA, J.; BULHÕES, L.J.; MOREIRA, J.A.A.; GRASSI FILHO, H. Correlação entre leituras de clorofila e níveis de nitrogênio aplicados em feijoeiro. Bragantia, v.55, p.171-175, 1996.

GILBER, A.; SILVA, P.R.F.; BORTOLINI, C.G. Clorofila na folha como indicador do nível de nitrogênio em cereais. Revista Ciência Rural, v.31, p.715-722, 2001.

MALAVOLTA, E.; VITTI, G.C.; OLIVEIRA, A.S. Avaliação do estado nutricional das plantas: princípios e aplicações. Piracicaba, POTAFÓS. 319p. 1997.

MARTINEZ, H.E.P.; CARVALHO J.G.; SOUZA R.B. Diagnose foliar. In: RIBEIRO AC, GUIMARÃES PT \& ALVAREZ VH (Eds). Recomendações para o uso de corretivos e fertilizantes em Minas Gerais $5^{\mathrm{a}}$ aproximação. Viçosa. p.143-168. 1999.

PIEKIELEK, W.P.; FOX, R.H. Use of a chlorophyll meter to predict sidedress nitrogen requirements for maize. Agronomy Journal, v.84, p.59-65, 1992.

REGAZZI, A.J. Modelos de regressão não linear e aplicações utilizando o SAS (PROC NLIN), 2 ed. Viçosa, UFV 131p. 2000.

ROSOLEM, C.A.; MARUBAYASHI, O.M. Seja o doutor do seu feijoeiro. Encarte do Informações Agronômicas - n. 68 - dezembro, 1994. Disponível em: <http://www.potafos.org/ppiweb/brazil.nsf/> Acesso em: 14 de outubro de 2007.

SILVEIRA, P.M.; BRAZ, A.J.B.P.; DIDONET, A.D. Uso do clorofilômetro como indicador da necessidade de adubação nitrogenada em cobertura no feijoeiro. Pesquisa Agropecuária Brasileira, n.38, p.1083-1087, 2003. 